\title{
Long-term follow-up after catheter ablation for atrioventricular nodal reentrant tachycardia: a comparison of cryothermal and radiofrequency energy in a large series of patients
}

\author{
Bruno Schwagten • Paul Knops • Petter Janse • \\ Geert Kimman • Yves Van Belle • Tamas Szili-Torok • \\ Luc Jordaens
}

Received: 16 September 2010 / Accepted: 8 November 2010 /Published online: 14 December 2010

(C) The Author(s) 2010. This article is published with open access at Springerlink.com.

\begin{abstract}
Background Radiofrequency (RF) catheter ablation for atrioventricular nodal reentrant tachycardia (AVNRT) is highly successful but carries a risk for inadvertent atrioventricular block. Cryoablation (cryo) has the potential to assess the safety of a site before the energy is applied.

Purpose The aim of this study was to evaluate the longterm efficacy and safety of cryothermal ablation in a large series of patients and compare it to RF.

Methods All consecutive routinely performed AVNRT ablations from our centre between 1999 and 2007 were retrospectively analysed.

Results In total, 274 patients were elegible: 150 cryoablations and $124 \mathrm{RF}$. Overall procedural success was 96\% (262/274), and equal in both groups, but nine patients were crossed to another arm. Mean fluoroscopy time was longer in the group treated with RF $(27 \pm 22$ min vs. cryo $19 \pm$ $15 \mathrm{~min} ; p=0.002)$. Mean procedure time was not different (RF $138 \pm 71 \mathrm{~min}$ vs. cryo $146 \pm 60 \mathrm{~min}$ ). A permanent pacemaker was necessary in two RF patients. The ques-

B. Schwagten • P. Knops • P. Janse • G. Kimman • Y. Van Belle •

T. Szili-Torok $\cdot$ L. Jordaens

Department of Cardiology,

Thoraxcenter, Erasmus Medical Center,

Rotterdam, The Netherlands

L. Jordaens

e-mail: 1.jordaens@erasmusmc.nl

B. Schwagten $(\square)$

Department of Electrophysiology,

Thoraxcenter, Erasmus Medical Center,

's Gravendijkwal 230,

3015 CE, Rotterdam, The Netherlands

e-mail: Brunoschwagten@hotmail.com
\end{abstract}

tionnaire revealed a high incidence of late arrhythmia related symptoms (48\%), similar in both groups, with improved perceived quality of life. The number of redo procedures for AVNRT over $4.3 \pm 2.5$-years follow-up was not statistically different (11\% after cryo and 5\% after RF). Conclusions Our data confirm that cryo and RF ablation with 4-mm tip catheters for AVNRT are equally effective, even after long-term follow-up.

Keywords Arrhythmias · Atrioventricular block · Atrioventricular reentrant tachycardia - Catheter ablation . Cryoablation $\cdot$ Radiofrequency

\section{Introduction}

Catheter ablation is a successful and widely used approach for treatment of atrioventricular reentrant tachycardia (AVNRT). [1, 2] However, there is still debate about the choice of the most optimal energy. Radiofrequency (RF) catheter ablation is highly effective but carries the risk for creating inadvertent atrioventricular heart block (AVB). [3] Cryoablation (cryo) on the other hand can be performed very safely due to the possibility of 'ice mapping' and 'cryoadherence'. [4] We showed in the past in a randomised trial that both techniques had the same acute outcome. [5] However, conflicting data exist about the recurrence rate with this technique, ranging from $0 \%$ to more than $30 \%$. [6-13] In this work, we evaluated not only the short-term efficacy and safety of RF ablation and cryothermal ablation of AVNRT in a large series of patients, but also the long-term outcome. 


\section{Methods}

All catheter ablations performed at our centre from 24 February 1999 to 19 December 2007 were analysed. In all procedures, the antiarrhythmic agents were discontinued for at least five half-lives before the procedure. The ablation protocols were unchanged during the whole inclusion period, and were supervised by the same electrophysiologist. However, the team consisted of several experienced electrophysiologists, with their own training and preferences. All procedures were performed under local anaesthesia, implemented with sedation. The following data were collected: energy used, acute success, number of applications, ablation time, fluoroscopy time, procedural time, acute and late complications and need for redo procedures. Procedural time was measured from the time of puncturing the vein until the time of removal of the sheaths from the groin, including the 30 -min waiting period after a successful ablation. The patients included in two previously published studies were not a part of this retrospective analysis. [5, 14]

\subsection{Ablation procedure}

One bipolar catheter, one quadripolar and one decapolar catheter were inserted to perform a standard electrophysiological study. The catheters were advanced into the heart and positioned respectively in the right ventricular apex, on the His bundle and in the coronary sinus. At baseline, the $\mathrm{AH}$ and $\mathrm{HV}$ intervals were recorded on the catheter in the His position, and with programmed electrical stimulation the presence of dual AV conduction was confirmed, defined as a AH jump of more than $50 \mathrm{~ms}$. Subsequently, tachycardia was induced by programmed electrical stimulation or by atrial burst pacing. If sustained, tachycardia could not be induced, isoprenaline was used. Induction of AVNRT was performed at least two times before moving forward to ablation.

Cryoablation Cryoablation was performed by using a Freezor 3 catheter with a 4-mm tip (Cryocath Technologies, Montreal, Canada) and a CCT2 CryoConsole (Cryocath Technologies, Montreal, Canada). At the site of interest, ice mapping was performed by cooling to $-30^{\circ} \mathrm{C}$ for a maximum of $60 \mathrm{~s}$. Fluoroscopy was applied until a stable temperature of $-30^{\circ} \mathrm{C}$ was reached. During the $60 \mathrm{~s}$, atrial extrastimulus testing was performed. Termination or noninducibility of AVNRT or disappearance of an AH-jump was considered to be an identification of a potentially successful ablation site. Ablation was subsequently performed using a freezing protocol by cooling to $-75^{\circ} \mathrm{C}$ for a 6-min period to create a permanent lesion. If there was no clear AH-jump, and AVNRT was difficult to induce at baseline, prolongation of the anterograde AV refractory period during atrial extra stimulus testing during ice mapping was used to identify a target site. The endpoint was non-inducibility of AVNRT. This was tested after each application, and if non-inducible, repeated after a 30-min waiting period.

$R F$ ablation $\mathrm{RF}$ catheter ablation was performed using a 4-mm, solid-tip, ablation catheter (Biosense-Webster Inc, Diamond Bar, CA, USA or an EPT Blazer, Boston Scientific, San Jose, CA, USA) in a temperature-controlled mode (maximum temperature $55^{\circ} \mathrm{C}$, maximum duration $60 \mathrm{~s}$, maximum $40 \mathrm{~W}$ ) with the use of a Stockert RF generator (BiosenseWebster Inc, Diamond Bar, CA, USA). Ablation of the slow pathway was guided by a combination of intracardiac electrograms and anatomical landmarks. When an optimal balance of a small fractionated $A$ and large $V$ signals were seen, RF energy was applied starting at a low energy level. Each application was started with the power set at $10 \mathrm{~W}$ and, if AV conduction was preserved, the power was progressively increased to $40 \mathrm{~W}$ until a maximum of $55^{\circ} \mathrm{C}$ was reached or junctional rhythm appeared. Fluoroscopy was used throughout each application. With both techniques, retrograde conduction was continuously monitored. The endpoint was non-inducibility of AVNRT. This was tested after each application, and if non-inducible, repeated after a 30-min waiting period.

\subsection{Follow-up}

Before hospital discharge, all patients received a 12-lead ECG recording and echocardiographic examination. Three months after the procedure all patients were examined at the outpatient clinic and asked about recurrent palpitations. If longstanding palpitations occurred within the follow-up period, a new 24-h holter monitor was performed or an effort was made to document the arrhythmia. Patients were then sent to their referring cardiologist, except when a redo procedure was necessary. For the purpose of this study, all patients were again personally approached by the investigators at the beginning of 2008 with a dedicated questionnaire, focusing on recurrence, duration and intensity of arrhythmias, antiarrhythmic drug use, cardioversion, readmission for cardiovascular reasons, for pacemaker implantation or reablation, also for other arrhythmias. This questionnaire was also sent to patients included in former trials as the obtained results were supposed not to be dependent on acute procedural outcomes [5, 14].

\subsection{Statistical analysis}

Continuous variables were expressed as mean \pm standard deviation, if normally distributed, or otherwise by median. 
The Students $t$ test or analysis of variance was used when appropriate. A Chi-square test with Yates correction was used for categorical data. The level of significance was set at $p<0.05$. For efficacy and complications analysis, therapy as applied. All statistics were performed using SPSS (16.0) for Windows (Chicago, Il, USA).

\section{Results}

\subsection{Patient data}

Of the 389 consecutive procedures performed at our centre between 24 February 1999 and 19 December 2007, 81 were excluded as they were already part of other published studies $[5,14]$. Of the remaining 308 procedures, 16 were excluded because another arrhythmia was identified during the study before ablation, and 17 others because of insufficient data (Fig. 1). Patients in whom AVNRT was ablated along with another arrhythmia (atrial flutter or atrial tachycardia) and redo procedures (28, of which 11 with cryo and 17 with RF) remained included. Eighty-eight (32\%) of 274 patients were male. The overall mean age was $51 \pm 17$ years, $50 \pm 17$ in the cryo group vs. $52 \pm 17$ years in the RF group (NS).

\subsection{Procedure and ablation data}

The initial approach was cryoenergy for 150 and RF for 124 ablations ( $55 \%$ vs. $45 \%)$. The overall acute success rate was $95 \%$ (262 patients). Of these procedures, 246 were a first ablation for AVNRT, and 28 were redo procedures. Only 10/274 patients (3.6\%) would fail the ablation (five in each group had procedural failure), but this implied a crossover of eight cryo patients to RF, and one RF to cryo. The

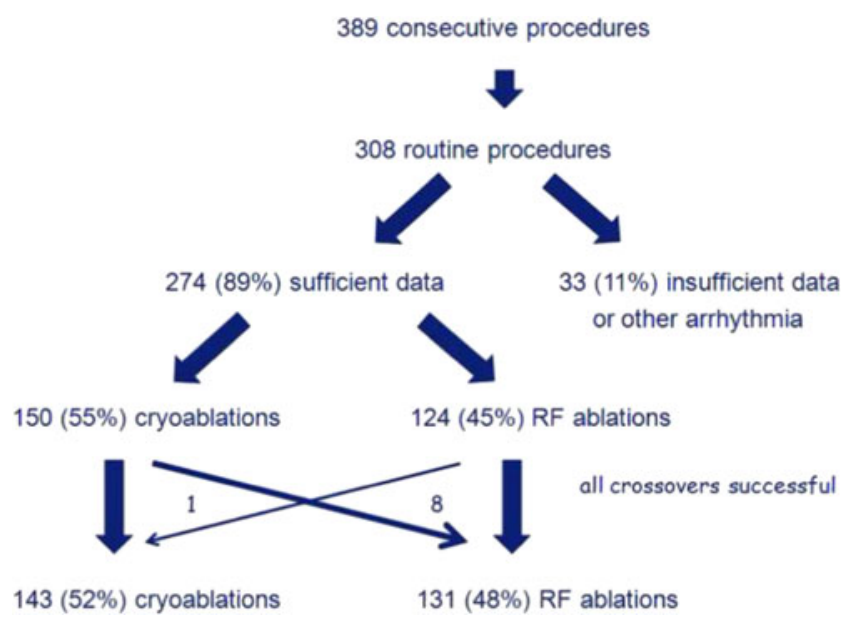

Fig. 1 Patient flow chart. At the beginning, study patients were excluded, resulting in 308 routine procedures. $A V N R T$, atrioventricular nodal reentrant tachycardia; $R F$, radiofrequency acute failure rate for the final delivered energy modality was similar for both approaches (cryo $3 \%$ vs. RF $4 \%$; NS). The median number of applications was less in the cryo group (two vs. six; Table 1). The mean ablation time was longer in the cryo patients $(522 \pm 384 \mathrm{~s}$ vs. $371 \pm 621 \mathrm{~s})$. The mean fluoroscopy time was shorter in the cryo group $(19 \pm 15 \mathrm{~min}$ vs. $27 \pm 22 \mathrm{~min}$ ), while the procedure time was not different (cryo $146 \pm 60 \mathrm{~min}$ vs. RF $138 \pm 71 \mathrm{~min}$ ). From the redo procedures, only one (cryo) failed acutely.

\subsection{Acute complications}

Thirteen patients had temporary AVB (six cryo vs. seven RF, including one cross-over patient; NS). Two patients had pericardial effusion needing acute intervention (both in the $\mathrm{RF}$ group). Further, two patients in the RF group received a permanent PM because of third degree atrioventricular block with narrow QRS complexes, without recovery before discharge.

\subsection{Long-term follow-up data}

After a mean follow-up of $4.3 \pm 2.5$ years, 20 of the 246 patients $(8 \%)$ treated for the first time in this series did undergo a redo ablation. The number of redo AVNRT procedures divided by the number of first procedures was not statistically different for both groups (14/132 for cryo vs. $6 / 114$ for RF). During three redo procedures the arrhythmia proved to be a left-sided concealed accessory pathway.

\subsection{Questionnaire}

The overall response rate to the questionnaire was $235 / 389$ (61\%). Of all patients responding to the questionnaire, $49 \%$ initially underwent cryo and 51\% RF ablation (NS). There was only one patient from the cross-over group to RF, and she is included in the RF group. Arrhythmia related symptoms were reported in 55/115 (47.8\%) in the cryo group versus 57/ $120(47.5 \%)$ in the RF group (NS). Table 2 displays the distribution of the arrhythmia pattern and the reported duration of the arrhythmias as reported in the questionnaire. Whereas the majority of the patients initially reported monthly or weekly occurrence of palpitations with a typical duration of hours, rather than minutes, this changed to no palpitations or sporadic occurrence, sometimes lasting only seconds after the procedure (Fig. 2). No differences were seen between the groups. The perceived quality of life improved in $102 / 115$ patients $(89 \%)$ in the cryo vs $106 / 120$ $(88 \%)$ in the RF group (NS; Fig. 3); 12/115 patients $(10 \%)$ in the cryo vs $12 / 120(10 \%)$ in the RF group (NS) observed no difference in their quality of life; only $1 / 115$ in the cryo and 2/120 in the RF group (NS) indicated a deterioration in perceived quality of life. From the 235 responses, 66 (28\%) 
Table 1 Procedural data

(analysis including cross-overs)

Analysis taking into account all patients as they were finally treated $A V B$ atrioventricular block; $N A$ not applicable; $n r$ number; $N S$ not significant; $R F$ radiofrequency; $S D$ standard deviation

\begin{tabular}{lccc}
\hline & Cryoablation & RF ablation & $p$ value \\
\hline Nr (final approach) & 143 & 131 & $<0.001$ \\
Application number (mean \pm SD); median & $2 \pm 22$ & $9 \pm 86$ & $<0.001$ \\
Ablation time (mean \pm SD); s & $522 \pm 384$ & $317 \pm 621$ & $<0.002$ \\
Fluoroscopy time (mean \pm SD); min & $19 \pm 15$ & $27 \pm 22$ & NS \\
Procedural time (mean \pm SD); min & $146 \pm 60$ & $138 \pm 71$ & NS \\
Acute success (nr) & $138 / 143$ & $126 / 131$ & NS \\
Temporary AVB (nr) & 6 & 7 & NA \\
Permanent AVB & 0 & $114 / 17$ & NA \\
First procedure/Redo & $132 / 11$ & & \\
\hline
\end{tabular}

patients reported a hospital readmission (Fig. 4). From the 35 arrhythmia-related admissions, 19 (54\%) were after cryo, 16 (46\%) after RF (NS). Two patients were admitted for elective cardioversion (atrial fibrillation). Five patients received a device for arrhythmia management. The two late ICD's were implanted after anterior myocardial infarction and after development of heart failure in a patient with nonischemic cardiomyopathy. Neither conduction disease nor arrhythmias were recorded in these two patients. The three late pacemaker indications were sinus node disease (one from each group), and one second-degree AVB with symptoms (RF group).

\section{Discussion}

The major finding of this study is that cryo and RF ablation for AVNRT are equally effective techniques, also in the long run. Cryoablation has a better safety profile.

Table 2 Reported arrhythmia pattern and duration (numbers) before and after ablation as answered in the questionnaire

\begin{tabular}{lllll}
\hline & \multicolumn{2}{l}{ Cryoablation $(N=115)$} & \multicolumn{2}{l}{$\mathrm{RF}(N=120)$} \\
\hline Arrhythmia pattern & Before & After & Before & After \\
None & 1 & 63 & 1 & 63 \\
Sporadic & 9 & 29 & 12 & 33 \\
Monthly & 40 & 15 & 43 & 12 \\
Weekly & 40 & 7 & 35 & 8 \\
Daily & 25 & 1 & 29 & 4 \\
Arrhythmia duration & Before & After & Before & After \\
None & 0 & 77 & 1 & 70 \\
Seconds & 2 & 18 & 3 & 23 \\
Minutes & 30 & 14 & 32 & 17 \\
Hours & 72 & 6 & 75 & 9 \\
Days & 11 & 0 & 9 & 1 \\
\hline
\end{tabular}

The pattern and duration before and after ablation are different $(p<$ $0.0001)$; no differences are observed after ablation between the two treatment modalities

$N$ number; $R F$ radiofrequency ablation

\subsection{Procedural efficacy}

Both cryo and RF ablation are very successful techniques in AVNRT ablation. Acute success was very high in previous studies, and this is confirmed in our observations, which were also achieved with a 4-mm catheter tip.[5-7] We cannot completely explain the relatively high cross-over rate, but we suspect that physician preference played a role in this. Use of a 6 -mm cryocatheter tip might improve the success rate, compared to a 4-mm tip, when applying on the right spot, but also might diminish the potential of ice mapping, as it yields larger lesions, and therefore might

\section{Reported arrhythmia pattern}

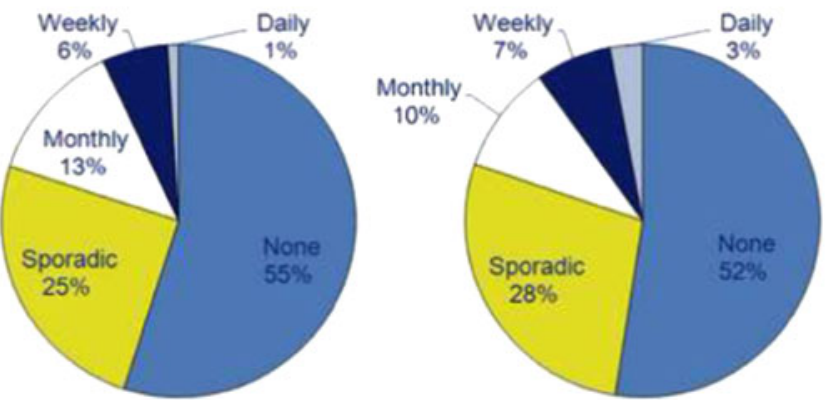

\section{Reported arrhythmia duration}

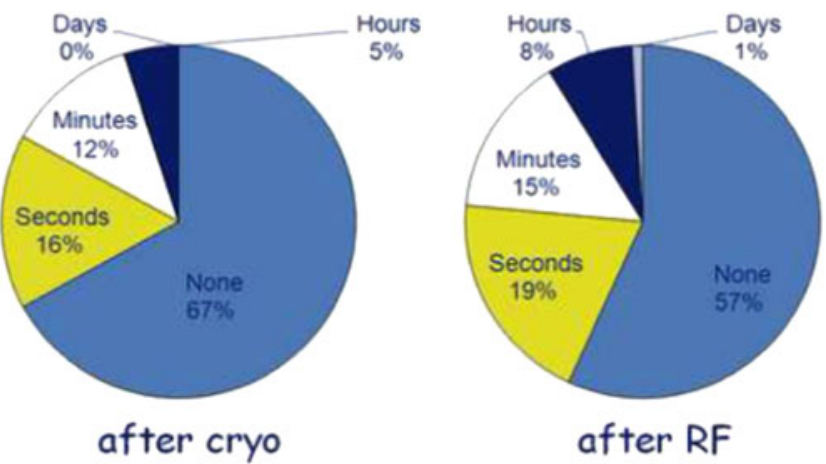

Fig. 2 Reported arrhythmia pattern and duration in the questionnaire $(N=235)$ after ablation (percentages) with cryoenergy (left) and RF (right). There are no differences in both parameters for the two approaches 


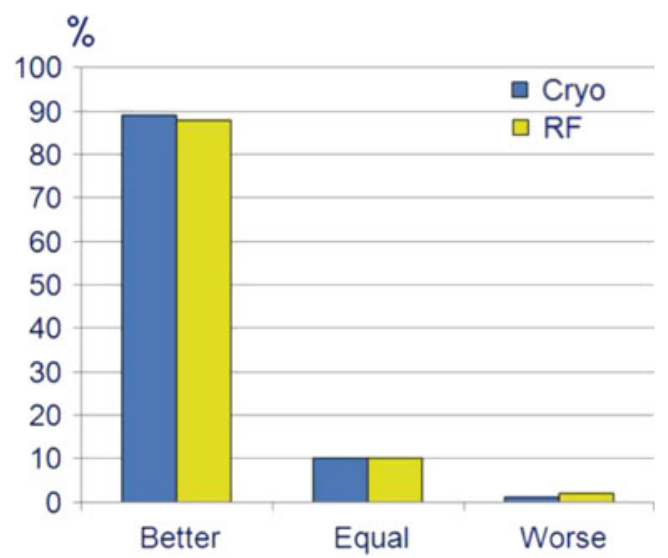

Fig. 3 Percentage of patients indicating an improvement, no difference or deterioration in their quality of life before and after the cryoablation (cryo) or radiofrequency $(R F)$ ablation

result in false-positive conduction damage so that no applications are given.[8-13]

The technique of RF ablation is well known: anatomical and electrical landmarks guide the procedure aimed to eliminate the slow pathway. The procedure is associated with some difficulties as the RF catheter tip moves during ablation due to changes in heart rhythm, respiratory movements and potential patient movements. For these reasons and also because of potential anatomic variations, damage to the fast pathway can occur in the triangle of Koch which is very small in comparison to a relatively large RF lesion. The need for careful imaging during RF ablation, which is not necessary during cryoablation, explains the shorter fluoroscopy time in our hands, confirming the data from other studies. $[5,6,10]$

\subsection{Safety}

The major potential advantage of cryoablation is its safety in preserving normal atrioventricular conduction. The ablation lesions formed by cryomapping probably always are reversible. Block during mapping at minus $30^{\circ} \mathrm{C}$, is a warning that no application should be given at this site [4]. However, even with short freezes at minus $75^{\circ} \mathrm{C}$ on the compact $\mathrm{AV}$ node or on the His, the
Fig. 4 Hospital admissions according to the questionnaire. $A V B$, atrioventricular block; $P M$, pacemaker; $S S S$, sick sinus syndrome; ICD, implantable cardioverter defibrillator
389 questionnaires sent<smiles>C=C[Te]</smiles>

235 responded (61\%)

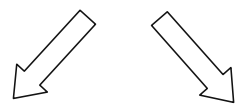

66 hospital readmission (28\%)

169 no hospital readmission (72\%)<smiles>C=CC=C</smiles>

35 arrhythmia related admissions

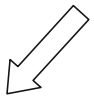

19 after cryo (16.5\%)<smiles>[CH]1C=C1</smiles>

$1 \mathrm{PM}$ for SSS

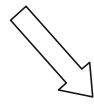

16 after RF (13.3\%)<smiles>C=CC=C</smiles>

$1 \mathrm{PM}$ for SSS

1 PM for second degree AVB

1 ICD after anterior myocardial infarction with heart failure

1 ICD in non-ischemic cardiomyopathy 
conduction seems to recover spontaneously almost always after waiting a short time [8]. With both the cryo and RF approach temporary heart block occurred in a similar amount. Further, we have seen unexpected temporary AVB occurring during real cryoablation, after cryomapping without blocking, but have never met inadvertent persisting heart block after such a procedure [5]. In contrast, in spite of a well-trained group of electrophysiologists, we observed two times complete AVB requiring permanent pacing in the RF group, and detected one additional late conduction problem after RF.

\subsection{Symptoms versus success}

Although cryo and RF have very high acute and longterm success rates, a significant amount of patients still have some arrhythmia-related complaints as shown by the questionnaire. Nevertheless, most patients indicate an improvement in quality of life after the ablation. It is well known that palpitations after AVNRT ablation often bear no relationship with the original arrhythmia: some patient complain of sinus tachycardia, atrial premature beats, ventricular premature beats or have an associated arrhythmia [3, 15]. Moreover, after AVNRT ablation atrial flutter might become present, or ectopic atrial tachycardia may arise due to the scarring [3]. It also has to be said when interpreting these results that atrial or ventricular premature beats, which often initiate AVNRT, are not treated by ablation of the slow pathway and may continue to provoke symptoms in both groups. An association with ventricular outflow tachycardia has been described, and is not uncommon [16-18]. Finally, it is known that in the long run, supraventricular tachycardia is associated with the development of atrial flutter or fibrillation. This indicates that judging the success of the AVNRT procedure merely by relying on symptoms is inefficient and can lead to incorrect conclusions. [15]

\subsection{Late recurrence}

Our study suggests that cryo and RF have a similar early and late failure rate, as reflected by the amount of redo procedures, even after a mean follow-up of over more than 4 years. When cryo is used on an intelligent way (i.e. with conventional pacemapping at $-30^{\circ} \mathrm{C}$ ), its success rate is equal to RF ablation in AVNRT (even when using a 4-mm tip). We always use pacing during cryomapping to prove that the AH-jump disappears, to see non-inducibility of AVNRT, or when cryomapping is performed during tachycardia, to witness termination of the AVNRT. Only after fulfilling at least one of the aforementioned criteria we proceed to cryoablation.

\subsection{Limitations}

The major limitation of this study is its retrospective design. However, the early results are in line with all former trials trying to compare cryo with $\mathrm{RF}$ in a randomised fashion, (including our own study) or in a retrospective way [5-13]. Further, the high cross-over rate created a discrepancy between the number intended to be treated with one approach, and the number really treated with one or another approach. However, this minor shift in numbers was taken into account in the interpretation of the data, so that the finally used energy modality took the benefit and the disadvantage.

The value of the questionnaire can be debated, as no standardised instrument to measure quality of life was used, and as no questionnaire was taken before the ablation. [19] On the other hand, patients indicated a perception of a better quality of life, indicating that some positive change occurred in a large majority after the treatment.

\subsection{Conclusion}

The overall success rate is very high and similar for both techniques. There is more need for fluoroscopy in the RF group, but procedures are equal in duration. There was no need to implant any pacemaker after cryoablation. Our data confirm that cryo and RF ablation for AVNRT are equally efficient, even after long-term follow-up, but still are associated with a high hospital admission rate during follow-up.

Disclosures Luc Jordaens was consultant for Cryocath during the time of this study.

Open Access This article is distributed under the terms of the Creative Commons Attribution Noncommercial License which permits any noncommercial use, distribution, and reproduction in any medium, provided the original author(s) and source are credited.

\section{References}

1. Haissaguerre, M., Warin, J. F., Lemetayer, P., Saoudi, N., Guillem, J. P., \& Blanchot, P. (1989). Closed-chest ablation of retrograde conduction in patients with atrioventricular nodal reentrant tachycardia. The New England Journal of Medicine, 320, 426433.

2. Jazayeri, M. R., Hempe, S. L., Sra, J. S., Dhala, A. A., Blanck, Z., Deshpande, S. S., et al. (1992). Selective transcatheter ablation of the fast and slow pathways using radiofrequency energy in patients with atrioventricular nodal reentrant tachycardia. Circulation, 85, 1318-1328.

3. Kimman, G. P., Bogaard, M. D., van Hemel, N. M., van Dessel, P. F., Jessurun, E. R., Boersma, L. V., et al. (2005). Ten year followup after radiofrequency catheter ablation for atrioventricular nodal reentrant tachycardia in the early days forever cured, or a source 
for new arrhythmias? Pacing and Clinical Electrophysiology, 28, 1302-1309.

4. Dubuc, M., Roy, D., Thibault, B., Ducharme, A., Tardif, J. C., Villemaire, C., et al. (1999). Transvenous catheter ice mapping and cryoablation of the atrioventricular node in dogs. Pacing and Clinical Electrophysiology, 22, 1488-1498.

5. Kimman, G. P., Theuns, D. A., Szili-Torok, T., Scholten, M. F., Res, J. C., \& Jordaens, L. J. (2004). CRAVT: a prospective, randomized study comparing transvenous cryothermal and radiofrequency ablation in atrioventricular nodal re-entrant tachycardia. European Heart Journal, 25, 2232-2237.

6. Zrenner, B., Dong, J., Schreieck, J., Deisenhofer, I., Estner, H., Luani, B., et al. (2004). Transvenous cryoablation versus radiofrequency ablation of the slow pathway for the treatment of atrioventricular nodal re-entrant tachycardia: a prospective randomized pilot study. European Heart Journal, 25, 2226-2231.

7. Collins, K. K., Dubin, A. M., Chiesa, N. A., Avasarala, K., \& Van Hare, G. F. (2006). Cryoablation versus radiofrequency ablation for treatment of pediatric atrioventricular nodal reentrant tachycardia: initial experience with 4-mm cryocatheter. Heart Rhythm, $3,564-570$.

8. Gupta, D., Al-Lamee, R. K., Earley, M. J., Kistler, P., Harris, S. J., Nathan, A. W., et al. (2006). Cryoablation compared with radiofrequency ablation for atrioventricular nodal re-entrant tachycardia: analysis of factors contributing to acute and followup outcome. Europace, 8, 1022-1026.

9. Avari, J. N., Jay, K. S., \& Rhee, E. K. (2008). Experience and results during transition from radiofrequency ablation to cryoablation for treatment of pediatric atrioventricular nodal reentrant tachycardia. Pacing and Clinical Electrophysiology, 31, 454-460.

10. Chan, N. Y., Mok, N. S., Lau, C. L., Lo, Y. K., Choy, C. C., Lau, S. T., et al. (2009). Treatment of atrioventricular nodal re-entrant tachycardia by cryoablation with a $6 \mathrm{~mm}$-tip catheter vs. radiofrequency ablation. Europace, 11, 1065-1070.

11. De Sisti, A., Tonet, J., Barakett, N., Lacotte, J., Leclercq, J. F., \& Frank, R. (2007). Transvenous cryo-ablation of the slow pathway for the treatment of atrioventricular nodal re-entrant tachycardia: a single-centre initial experience study. Europace, 9, 401-406.

12. Sandilands, A., Boreham, P., Pitts-Crick, J., \& Cripps, T. (2008). Impact of cryoablation catheter size on success rates in the treatment of atrioventricular nodal re-entry tachycardia in 160 patients with long-term follow-up. Europace, 10, 683686.

13. Rivard, L., Dubuc, M., Guerra, P. G., Novak, P., Roy, D., Macle, L., et al. (2008). Cryoablation outcomes for AV nodal reentrant tachycardia comparing 4-mm versus 6-mm electrode-tip catheters. Heart Rhythm, 5, 230-234.

14. Thornton, A. S., Janse, P., Theuns, D. A., Scholten, M. F., \& Jordaens, L. J. (2006). Magnetic navigation in AV nodal re-entrant tachycardia study: early results of ablation with one- and threemagnet catheters. Europace, 8, 225-230.

15. Jordaens, L., Vertongen, P., \& Verstraeten, T. (1994). Prolonged monitoring for detection of symptomatic arrhythmias after slow pathway ablation in AV-nodal tachycardia. International Journal of Cardiology, 44, 57-63.

16. Kazemi, B., Arya, A., Haghjoo, M., \& Sadr-Ameli, M. A. (2006). Coincident atrioventricular nodal reentrant and idiopathic ventricular tachycardia. Asian Cardiovascular \& Thoracic Annals, 14, 284-288.

17. Haghjoo, M., Arya, A., Dehghani, M., Emkanjoo, Z., Fazelifar, A., Heidari, A., et al. (2005). Coexistence of atrioventricular nodal reentrant tachycardia and idiopathic left ventricular outflow-tract tachycardia. Indian Pacing Electrophysiology Journal, 5, 149-154.

18. Washizuka, T., Niwano, S., Tsuchida, K., \& Aizawa, Y. (1999). AV reentrant and idiopathic ventricular double tachycardias: complicated interactions between two tachycardias. Heart, 81, 318-320.

19. Arribas, F., Ormaetxe, J. M., Peinado, R., Perulero, N., Ramírez, P., \& Badia, X. (2010). Validation of the AF-QoL, a diseasespecific quality of life questionnaire for patients with atrial fibrillation. Europace, 12, 364-370. 